\title{
Sedimentation of Bidisperse, Uncharged Colloidal Sphere Suspensions: Influence of Viscosity and Irregular Surfaces
}

\author{
Dominique M. E. Thies-Weesie, Albert P. Philipse, and Henk N. W. LekKerkerker \\ Van't Hoff Laboratory for Physical and Colloid Chemistry, Utrecht University, Padualaan 8, P.O. Box 80.015, 3508 TB Utrecht, The Netherlands
}

Received March 1, 1995; accepted June 7, 1995

\begin{abstract}
The sedimentation velocity of uncharged, nonaggregated silica spheres under gravity is strongly reduced after addition of small amounts of nonsedimenting small spheres. This reduction is largely due to surface irregularities on a nanoscale of the large spheres at which a limited number of small spheres adsorbs, leading to an increase of the hydrodynamic friction per particle. This adsorption also screens effectively any weak $V$ an der $W$ aals attraction between the large spheres, which despite its weakness, significantly influences the concentration dependence of settling in a pure solvent. The concentration dependence and magnitude of the large-sphere sedimentation velocity in a more concentrated dispersion of small spheres agree with the prediction by Batchelor that the small particles mainly manifest themselves as an effective viscosity increase. @ 1996 Academic Press, Inc.
\end{abstract}

\section{INTRODUCTION}

The concentration dependence of the sedimentation velocity has been studied extensively theoretically and experimentally for monodisperse colloidal spheres $(1-4)$. For polydisperse spheres only the simplest case has been addressed, namely a bidisperse sphere mixture (5). Sedimentation results on micrometer-sized particle mixtures (6) are available, but experimental information on colloidal sphere mixtures is lacking. We designed an experiment in which large, uncharged colloidal silica spheres settle under influence of gravity in a dispersion of nonsedimenting small ones. The moving boundary of the turbid system of large particles can be clearly detected against the background of the small particles, which hardly scatter light. The silica particles are sterically stabilized by a grafted alkane layer and form stable dispersions in cyclohexane. Sedimentation experiments were performed on five silica dispersions and three mixtures of them to assess whether any system-dependent features, like interparticle attractions, are present.

For the silica systems under study, Batchelor's theory (5) makes the following predictions. If the small particles are small enough they will mainly manifest themselves as a modest viscosity increase of the medium in which the large particles now sediment more slowly. The concentration de- pendence of this sedimentation is expected to be the same as for monodisperse spheres in a "normal" solvent. For the hard spheres in Batchelor's theory this is indeed a plausible scenario. In practice, however, even weak interparticle attractions may complicate interpretation (and acquisition) of sedimentation results.

First, the small particles may induce a depletion attraction between the large spheres (7-9). This effect can be minimized by choosing sufficiently low particle concentrations. Second, there may be a significant Van der Waals attraction between the large spheres, because this attraction increases with the particle radius. Therefore it can be suppressed by decreasing the sphere size, though a compromise must be made because the spheres should be large enough to settle under influence of gravity.

But there are more attraction possibilities in a bidisperse system. In studies on the rheology (10), scattering properties (11), and phase separation (8) of binary silica sphere systems, indications for an (unexplained) attraction between large and small spheres were reported. Remarkably, the interactions in the separate monodisperse systems were clearly established to be purely repulsive. Our sedimentation experiments confirm the possibility of such an attraction between large and small silica particles. Supplementary experiments with, among other things, dynamic light scattering show that a small amount of small particles is adsorbed on the large spheres. This does not contradict the fact that the separate components do not stick. It is argued in Section 4.3. that surface roughness explains this peculiar phenomenon.

It turns out that a modest adsorption of small particles on - and fairly weak Van der Waals forces between-the large spheres significantly change their settling velocity. Nevertheless, for a sufficiently high concentration of free small particles, one expects to see, for example, Batchelor's viscosity effect.

The paper is organized as follows. In Section 2 Batchelor's equations for the sedimentation velocity in dilute mono- and bidisperse systems are briefly described. The synthesis and characterization of these particles and the experimental equipments can be found in Section 3. The sedimentation results of the mono- and bidisperse sphere systems and the 
comparison with Batchelor's theory are discussed in Section 4. Conclusions are summarized in Section 5.

\section{THEORY}

The sedimentation velocity, $U_{0}$, of a particle of arbitrary shape at infinite dilution is given by

$$
U_{0}=\frac{V_{\mathrm{p}}\left(\rho_{\mathrm{p}}-\rho_{0}\right) g}{f},
$$

where $V_{\mathrm{p}}$ is the particle volume, $\rho_{\mathrm{p}}$ the particle density, $\rho_{0}$ the solvent density, $g$ the gravitational acceleration, and $f$ the friction coefficient of the particle. The sedimentation velocity of a spherical particle of radius $a$, for which $V_{\mathrm{p}}=$ $(4 / 3) \pi a^{3}$ and Stokes' friction $f=6 \pi \eta_{0} a$, in a solvent with viscosity $\eta_{0}$ is

$$
U_{0}=\frac{2 a^{2}\left(\rho_{\mathrm{p}}-\rho_{0}\right) g}{9 \eta_{0}}
$$

At low volume fraction $\phi$ the sedimentation velocity $U$ of uncharged particles varies linearly with $\phi$ according to

$$
\frac{U}{U_{0}}=1-K \phi
$$

The constant $K$, which is mainly determined by backflow, is calculated by Batchelor (1) for monodisperse, hard spheres to be $K=6.55$. Experimentally the linearity in Eq. [3] is confirmed with values of $K$ varying between 5 and 7 (2-4).

Attractions between the particles cause in the vicinity of a test sphere an excess amount of particles, which profit from the downward motion of the test sphere. This reduces the average friction and consequently increases the average sedimentation velocity. Theoretically (1) and experimentally (12) it has been found that the value of $K$ for attractive particles is smaller than $K=6.55$ for hard spheres.

Long-range repulsive forces between sedimenting, charged particles not only change the value of $K$ in comparison with hard spheres, but, more importantly, change the linear relation in Eq. [3] into a nonanalytical $\phi$-dependence (13). In what follows only uncharged spheres are dealt with.

The sedimentation velocity of spheres of species $i$ through a dispersion of spheres of species $j$, depends on both the volume fractions $\phi_{i}$ and $\phi_{j}$. Batchelor (5) calculated the relevant coefficients $S_{i i}$ and $S_{i j}$ for bidisperse, hard spheres at low volume fractions,

$$
\frac{U_{i}}{U_{0}}=1+S_{i i} \phi_{i}+S_{i j} \phi_{j}
$$

Here $U_{0}$ is the single-particle sedimentation velocity of an $i$-sphere as is defined in Eq. [2] and $S_{i i}$ equals -6.55 in the absence of interparticle forces (5). $S_{i j}$ accounts for the hydrodynamic interactions due to the presence of the $j$ spheres. The expression of $S_{i j}$ is rather complicated (5). However, in the case of a small size ratio, $a_{j} / a_{i}, S_{i j}$ can be approximated by

$$
S_{i j} \simeq-\frac{5}{2}-\gamma
$$

where the particle density ratio $\gamma=\left(\rho_{j}-\rho_{0}\right) /\left(\rho_{i}-\rho_{0}\right)$. The factor 5/2 in Eq. [5] stems from the presence of small spheres $j$, producing an increase in the viscosity according to Einstein's relation $\eta=\eta_{0}(1+2.5 \phi)$ (14). The factor $\gamma$ partly originates from a retarding backflow induced by any sedimentation of small spheres $j$. The additional buoyancy force resulting from the greater density of the medium due to the presence of the $j$ spheres is also included in $\gamma$. All effects retard the sedimentation velocity of the large $i$ spheres. The size ratios of the three silica mixtures in this study are such that Eq. [5] applies. As an independent check of the viscosity effect we measured viscosities of smallsphere dispersions.

\section{EXPERIMENTAL}

\subsection{The Systems}

Three silica sphere systems, synthesized following Stöber's method (15), and two batches of commercially available ludox sphere systems (LUDOX HS-40, Stokvis), were coated with octadecylalcohol (Merck) to make them apolar $(16,17)$. The dispersions were purified from free octadecylalcohol by repeated centrifugation and redispergation in cyclohexane (Baker p.a.), which is a good solvent for the coated, uncharged particles. All Stöber silica spheres form rigid colloidal crystals upon sedimentation, which confirms that the systems consist of fairly monodisperse, nonaggregated particles. (Strong attractions between particles would prohibit crystallization.) The ludox spheres are too small to settle a measurable distance under gravity even on a time scale of weeks. In Table 1 the characterization results are summarized, which were obtained in the following way.

TABLE 1

Particle Characteristics

\begin{tabular}{lrcc}
\hline \multicolumn{1}{c}{ System } & \multicolumn{1}{c}{$a_{\text {TEM }}(\mathrm{nm})$} & $a_{\text {DLS }}(\mathrm{nm})$ & $\rho_{\mathrm{p}}\left(\mathrm{g} \mathrm{ml}^{-1}\right)$ \\
\hline SC150S & $115.9 \pm 6.4$ & 133.2 & $1.769 \pm 0.004$ \\
SD200S & $157.4 \pm 6.5$ & 182.4 & $1.688 \pm 0.007$ \\
SD250S & $229.9 \pm 9.7$ & 265.8 & $1.784 \pm 0.002$ \\
LUDOX2 & $7.7 \pm 1.9$ & 16.0 & $1.510 \pm 0.002$ \\
LUDOX3 & $7.3 \pm 1.4$ & 18.4 & $1.624 \pm 0.017$ \\
\hline
\end{tabular}



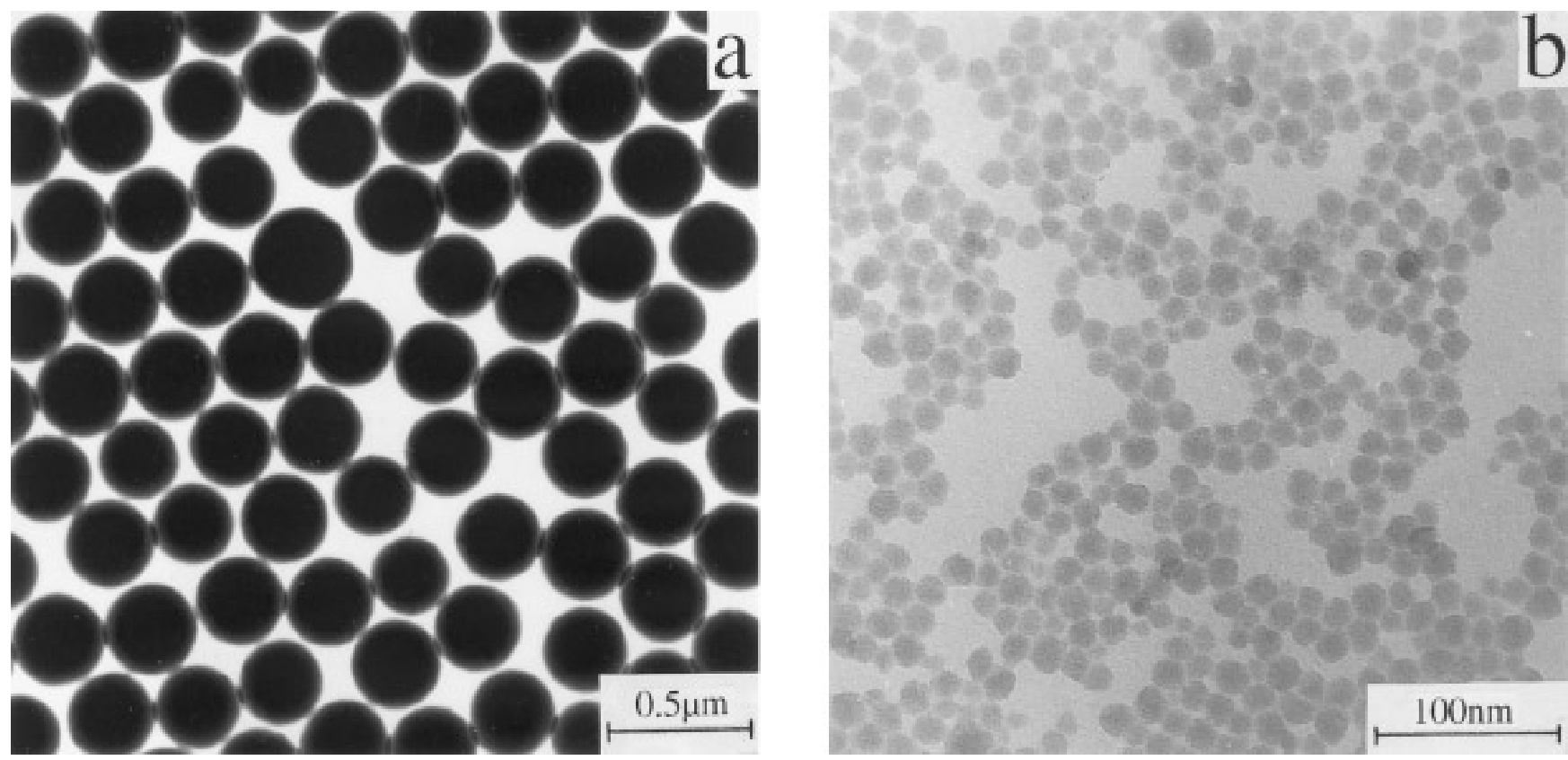

FIG. 1. Transmission electron micrographs of (a) SD200S spheres and (b) LUDOX3 particles. The large silica spheres are monodisperse in comparison with the small ludox particles (see Table 1).

Dynamic light scattering (DLS) was done on very dilute, dust-free dispersions with an argon laser (Spectra Physics Series 2000) operating at $488.0 \mathrm{~nm}$. Autocorrelation functions were measured with a Malvern Multibit K7025 128 points correlator at scattering angles between $35^{\circ}$ and $140^{\circ}$. From a second-order cumulant fit of the autocorrelation functions diffusion coefficients were obtained from which the hydrodynamic radius $a_{\text {DLS }}$ was calculated using the StokesEinstein equation

$$
a_{\mathrm{DLS}}=\frac{k_{\mathrm{B}} T}{6 \pi \eta_{0} D_{0}}
$$

where $k_{\mathrm{B}}$ is the Boltzmann constant, $T$ is the temperature, and $D_{0}$ is the free-particle diffusion coefficient.

Transmission electron microscopy (TEM) was performed on a Philips CM10 electron microscope. A carrier grid was dipped in a dilute dispersion and dried. From the obtained micrographs the average radius $a_{\mathrm{TEM}}$ of the spheres and its standard deviation were determined. Characteristic micrographs for the silica and ludox spheres are given in Fig. 1. The surface of the monodisperse spherical Stöber silica particles appears to be smooth at this magnification (see, however, Fig. 8a). The small ludox particles are somewhat polydisperse, and have a more irregular shape.

A weighted amount of dispersion of known mass density was dried at $80^{\circ} \mathrm{C}$ under a weak nitrogen flow to constant weight to obtain the mass fraction. With the solvent mass density the mass density $\rho_{\mathrm{p}}$ of the particles was calculated.
The volume fraction $\phi_{\mathrm{s}}$ of Stöber silica is $c / \rho_{\mathrm{p}}$, where $c$ is the silica weight concentration. This volume fraction equals the volume of silica $\left(V_{\mathrm{s}}\right)$ divided by the total dispersion volume $\left(V_{\text {disp }}\right)$. The volume fraction $\phi_{1}$ of ludox in a mixture with the large spheres is defined as

$$
\phi_{1}=\frac{V_{1}}{V_{\text {disp }}-V_{\mathrm{s}}}
$$

where $V_{1}$ is the total volume of ludox particles. So, different amounts of silica settle in the same background dispersion of ludox spheres in cyclohexane with the same dispersion viscosity $\eta$.

\subsection{Rheology}

Viscosity measurements on dilute dispersions of LUDOX3 in cyclohexane were performed using an Ubbelohde capillary viscometer (Schott Geräte) in which the time is measured for $5 \mathrm{ml}$ of dispersion to flow through a capillary (of $0.46 \mathrm{~mm}$ diameter) under gravity. The product of this time and the density of the sample is proportional to the viscosity. Because of the strong temperature dependence of the viscosity the capillary is placed in a thermostat at $T$ $=21.5^{\circ} \mathrm{C}$. After measuring the flow time of the solvent cyclohexane 10 times, the Ubbelohde was dried. A weighted amount of LUDOX3 dispersion was brought into the viscometer and the flow time of the sample was determined 10 times. The sample was diluted with a weighted amount of cyclohexane and after homogenization the flow time was 
measured again. This procedure of diluting and measuring was repeated another five times to find the relation between the viscosity $\eta$ and the ludox volume fraction $\phi_{1}$.

\subsection{Sedimentation}

Because the small ludox spheres do not sediment significantly under gravity an analytical ultracentrifuge (Beckman, model E) with a photo-electric scan system was used to determine the sedimentation velocity as a function of the volume fraction $\phi_{1}$ of LUDOX3. The temperature was kept constant at $21.5 \pm 0.1^{\circ} \mathrm{C}$. The samples were prepared by mixing weighted amounts of LUDOX3 dispersion and cyclohexane.

The sedimentation velocity of the large silica spheres, which is too large for the ultracentrifuge, was measured in a simpler equipment. Weighted amounts of silica in cyclohexane were poured in glass sedimentation tubes of $1 \mathrm{~cm}$ diameter and $15 \mathrm{~cm}$ length, which were carefully closed. Sedimentation is extremely sensitive to temperature changes and vibrations. They cause convections which complicate the analysis of sedimentation velocities. To prevent convections the sedimentation tubes were immersed in a water bath, which stood on a heavy, marble table. The equipment was placed in a thermostated room to keep the temperature constant at $21.5 \pm 0.1^{\circ} \mathrm{C}$. The height of the dispersion-solvent interface was followed for $24 \mathrm{~h}$ with a cathetometer consisting of a Zeiss Ni-40 leveling instrument coupled to a linear scale system (Mitutoyo AT-11-N), which allows measurements with an uncertainty of $0.01 \mathrm{~mm}$. To increase the visibility of the interfaces, the sedimentation tubes were illuminated with a slide projector. After $24 \mathrm{~h}$ the dispersions were homogenized by shaking and applying a Vortex-mixer and an ultrasonic bath for about $1 \mathrm{~min}$. Each sample was measured four times and the resulting sedimentation velocities, all converted to the same temperature $T=21.5^{\circ} \mathrm{C}$, were averaged.

After these four measurements weighted amounts of ludox dispersion were added to each of the tubes such that the ludox concentration is the same in every tube. The mixtures of dispersions were homogenized and the sedimentation procedure was repeated, after which again ludox was added. Mixtures were made of SC150S/LUDOX2, SD200S/LUDOX2, and SD250S/LUDOX3. The volume fraction of silica, $\phi_{\mathrm{s}}$, varied between 0.01 and 0.10 and the volume fraction of ludox, $\phi_{1}$, between 0.005 and 0.06 .

\section{RESULTS AND DISCUSSION}

\subsection{Monodisperse Systems}

4.1.1. Stöber silica spheres. The normalized sedimentation velocity $U_{\mathrm{s}} / U_{0}$ of the three Stöber silica systems as a function of the silica volume fraction $\phi_{\mathrm{s}}$ is shown in Fig. 2. The values of $U_{0}$ are determined from extrapolation of $U_{\mathrm{s}}$ to infinite dilution. The extrapolated values agree with the Stokes velocity, calculated from the particle characteristics, as can be seen in Table 2. For this calculation the hydrodynamic radius $a_{\text {DLS }}$ is used. This agreement shows that the silica particles do not stick together and that they sediment as separate spheres. The correspondence between theory and experiments also demonstrates that the followed sedimentation procedure (Section 3.3) is reliable and that possible convections do not (measurably) disturb the sedimentation process.

The value for the slope $K$ (in Eq. [3]) for the three silica systems in Fig. 2 is determined from a linear regression analysis for $\phi_{\mathrm{s}} \leqslant 0.06$ and is also given in Table 2 . For the smallest silica spheres, SC150S, a value of $K=6.2 \pm 0.4$ is obtained, which does not differ significantly from the value of $K=6.55$ calculated by Batchelor for monodisperse, hard spheres. This correspondence indicates that these spheres are not subjected to a significant net attraction or any other repulsion than the hard-sphere potential. For the two systems with larger silica spheres, SD200S and SD250S, a value of $K=3.0 \pm 0.2$ is found, which is probably caused by Van der Waals attractions between the particles. The Van der Waals attraction between two spheres of radius $a$ at a surface-to-surface distance $H$ is (18)

$$
V=-\frac{A}{6}\left[\frac{2}{s^{2}-4}+\frac{2}{s^{2}}+\ln \left(\frac{s^{2}-4}{s^{2}}\right)\right],
$$

where $s$ is a dimensionless distance, $s=(2 a+H) / a$, and $A$ is the Hamaker constant, which at room temperature has a value for silica in cyclohexane of $A \approx 0.15 k_{\mathrm{B}} T$ (19). The distance of closest approach of two stearyl-coated silica spheres is about $3 \mathrm{~nm}$, being approximately twice the thickness of the stearyl layer. Such a distance results in a contact attraction for the SD200S and SD250S spheres of respectively $-0.7 k_{\mathrm{B}} T$ and $-1.1 k_{\mathrm{B}} T$. This is comparable to the thermal energy, so the spheres do not permanently stick together. The weakness of the attraction also follows from the agreement between the experimental and theoretical values of $U_{0}$. (For example, permanent doublets would increase $U_{0}$ ). Further, in both systems (just as for SC150S) the spheres in the sediments form colloidal crystals. This confirms that attractions are fairly weak, because strong attractions produce an amorphous structure. Batchelor (1) derived for short-range attractions an extension of Eq. [3], which accounts for the influence of an excess amount of spheres at a distance $2 a$ from the test sphere on the sedimentation velocity of the test sphere. With this equation it is possible to relate the obtained value for $K$ with the interaction pair potential. The Van der Waals attraction in Eq. [8], however, cannot be modeled by a narrow attraction well, because it 

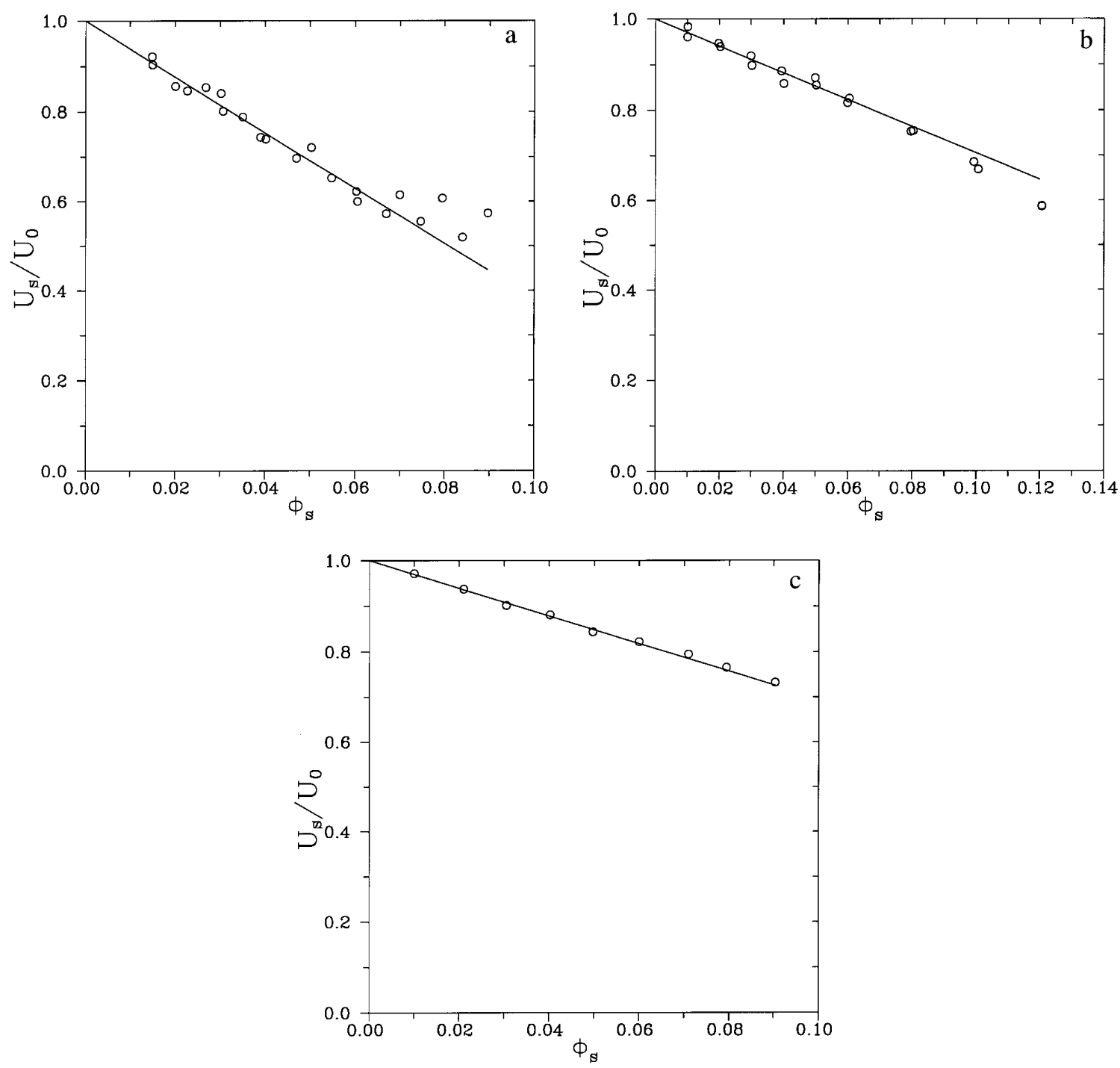

FIG. 2. Normalized sedimentation velocity $U_{\mathrm{s}} / U_{0}$ versus the volume fraction $\phi_{\mathrm{s}}$ for the large silica spheres in cyclohexane, for (a) SC150S, (b) SD200S, and (c) SD250S. The solid line is a best fit to Eq. [3] for $\phi_{\mathrm{s}} \leqslant 0.06$ and the values for $U_{0}$ and $K$ can be found in Table 2 .

acts over longer distances. The conclusion here is that small attractions significantly reduce $K$, but that its diminished value of $K=3.0$ is difficult to explain in terms of Batchelor's theory.

TABLE 2

Calculated and Measured $V$ alues for $U_{0}$ and $K$ in Eq. [3] for the Silica Systems and a Ludox System in Cyclohexane at $T=$ $21.5^{\circ} \mathrm{C}$

\begin{tabular}{lccr}
\hline System & $\begin{array}{c}\text { Calculation of } \\
U_{0}\left(\mathrm{~m} \mathrm{~s}^{-1}\right)\end{array}$ & $\begin{array}{c}\text { Measurement of } \\
U_{0}\left(\mathrm{~m} \mathrm{~s}^{-1}\right)\end{array}$ & $\begin{array}{c}\text { Measurement } \\
\text { of } K\end{array}$ \\
\hline SC150S & $(4.02 \pm 0.45) \times 10^{-8}$ & $(4.44 \pm 0.06) \times 10^{-8}$ & $6.2 \pm 0.4$ \\
SD200S & $(6.92 \pm 0.57) \times 10^{-8}$ & $(7.37 \pm 0.06) \times 10^{-8}$ & $3.0 \pm 0.2$ \\
SD250S & $(16.25 \pm 0.14) \times 10^{-8}$ & $(15.76 \pm 0.07) \times 10^{-8}$ & $3.0 \pm 0.1$ \\
LUDOX3 & $(6.55 \pm 2.59) \times 10^{-10}$ & $(4.71 \pm 0.07) \times 10^{-10}$ & $10.5 \pm 0.5$ \\
\hline
\end{tabular}

4.1.2. Ludox spheres. The sedimentation results of the LUDOX3 spheres are given in Fig. 3a and in Table 2. The experimental and theoretical values for $U_{0}$ coincide within the uncertainty in the calculated Stokes velocity, which is due to the large size polydispersity of the LUDOX3 particles. The choice for $U_{0}$ has consequences for the value of the slope $K$ ( see Eq. [3]). From the experimental $U_{0}$ a value of $K=10.5 \pm 0.5$ is obtained, which is rather large. Using the calculated $U_{0}$, based on the average particle size, $K$ becomes $7.6 \pm 3.0$, which is close to Batchelor's predictions for hard spheres. (This comparison must be made with caution, because ludox particles are polydisperse and are not really spherical.)

The relative viscosity $\eta / \eta_{0}$ as a function of the volume fraction $\phi_{1}$ of LUDOX3 is presented in Fig. 3b. The best-fit solid line has a slope of $5.1 \pm 0.1$, which is large compared 

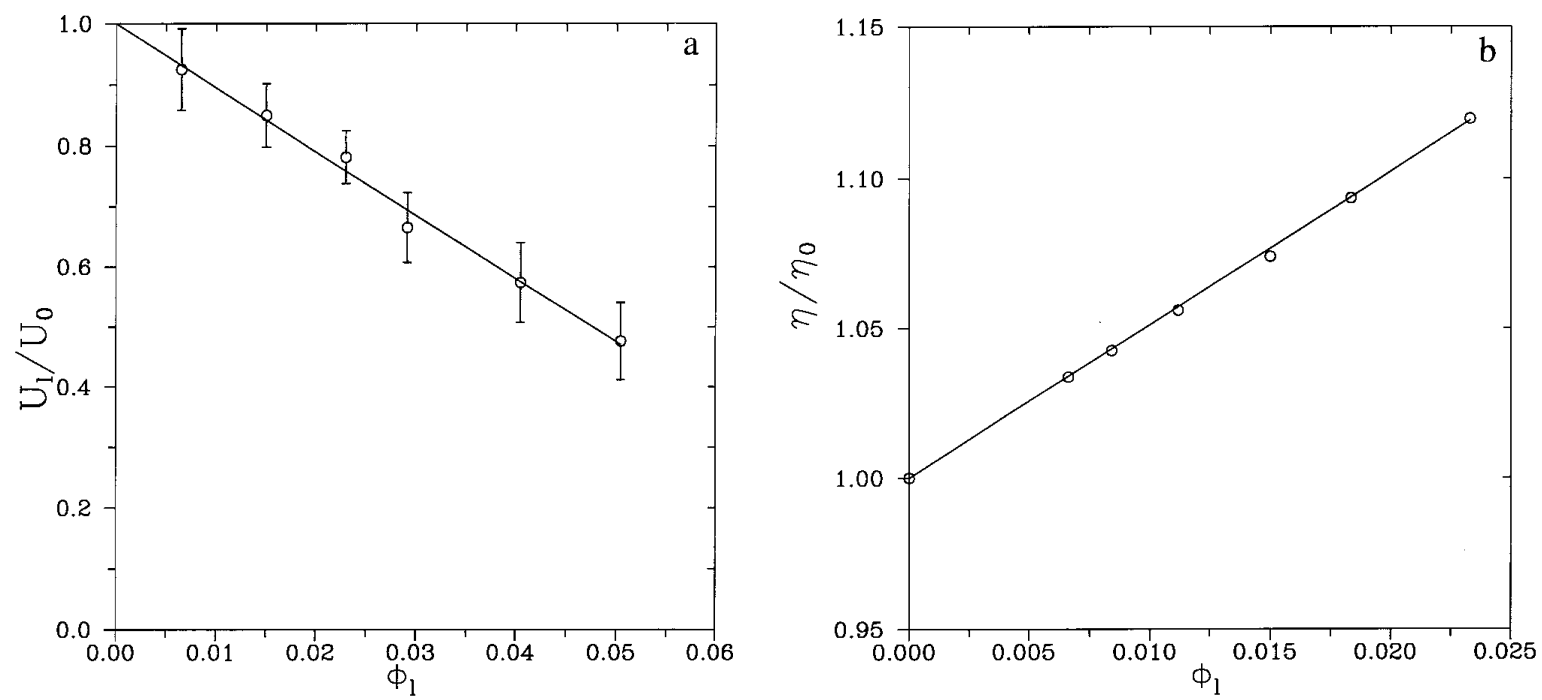

FIG. 3. (a) The sedimentation velocity $U_{1} / U_{0}$ as a function of $\phi_{1}$ for the small LUDOX3 particles in cyclohexane. The values for $U_{0}$ and for the slope $K$ are given in Table 2. (b) The relative viscosity $\eta / \eta_{0}$ is presented versus the volume fraction $\phi_{1}$ of LUDOX3. The solid line is a best fit and is given by $\eta / \eta_{0}=1+(5.1 \pm 0.1) \phi_{1}$.

with the intrinsic viscosity of 2.5 calculated by Einstein (14). This difference is caused by the fact that in our experiments values for $\phi_{1}$ were calculated from the mass density $\rho_{\mathrm{p}}$ of the dry particles, instead of the mass density of the particles in solution. In solution solvent molecules are immobilized between the alkyl chains at the silica surface, which enlarges the particle volume. So the "hydrodynamic"' particle volume is larger than expected on the basis of $\rho_{\mathrm{p}}$. Using the hydrodynamic particle volume in the calculation of $\phi_{1}$ decreases the value of the measured intrinsic viscosity.

\subsection{Bidisperse Systems}

The sedimentation velocity of Stöber silica spheres through a dispersion of (nonsedimenting) ludox spheres can be described at low volume fractions, analogous to Eq. [4], with

$$
\frac{U_{\mathrm{s}}}{U_{0}}=\left(1+S_{\mathrm{sl}} \phi_{\mathrm{l}}\right)-K \phi_{\mathrm{s}}=P-K \phi_{\mathrm{s}} .
$$

Here, the subscripts 1 and s denote respectively ludox and Stöber silica. For each silica-ludox mixture, the same experimental $U_{0}$ for silica is used, which is given in Table 2 . The changes in $P$ and in $K$, due to the addition of ludox to the silica dispersions, are discussed, respectively, in Sections 4.2.1. and 4.2.2.

4.2.1. The change in $P$. The first addition of ludox (see Table 3 ) to the three silica dispersions results in a marked reduction of $10-20 \%$ of the sedimentation velocity as can be seen in Fig. 4. From Batchelor's theory, Eqs. [4-5], only a $2-7 \%$ reduction of $P$ is expected (see Table 3 ). Such a modest reduction would account for the presence of the freely diffusing small spheres, which increase the viscosity and the buoyancy force and disturb the flow field of the large particles. The observed reduction is caused by an unexpected phenomenon, namely, the adsorption of ludox particles on the silica surface. The evidence for this adsorption is discussed in Section 4.3., where it is argued that the number of adsorbed ludox particles per silica sphere is in the range 10-60.

It is at first sight peculiar that coagulation of the small particles on the large silica spheres retards sedimentation. Usually particle coagulation increases sedimentation rates. This need not be the case, however, if particles considerably differ in size. The Stokes friction for a sphere with a surface

TABLE 3

Theoretical and Experimental Results for $P$ and $K$ in $U_{\mathrm{s}} / U_{0}=P-K \phi_{\mathrm{s}}$

\begin{tabular}{|c|c|c|c|c|}
\hline \multirow[b]{2}{*}{ System } & \multicolumn{2}{|c|}{$\begin{array}{c}\text { Theoretical } \\
\text { calculations } \\
\text { (5) }\end{array}$} & \multicolumn{2}{|c|}{ Experimental results } \\
\hline & $P$ & $K$ & $P$ & $K$ \\
\hline SC150S & 1 & 6.55 & 1 & $6.2 \pm 0.4$ \\
\hline SC150S/0.5\%LUDOX2 & 0.984 & 6.55 & $0.802 \pm 0.015$ & $3.1 \pm 0.3$ \\
\hline SC150S/1.0\%LUDOX2 & 0.968 & 6.55 & $0.765 \pm 0.014$ & $3.0 \pm 0.3$ \\
\hline SD200S & 1 & 6.55 & 1 & $3.0 \pm 0.2$ \\
\hline SD200S/2.0\%LUDOX2 & 0.934 & 6.55 & $0.785 \pm 0.013$ & $6.3 \pm 0.4$ \\
\hline SD200S/4.0\%LUDOX2 & 0.868 & 6.55 & $0.755 \pm 0.020$ & $6.1 \pm 0.6$ \\
\hline SD200S/6.0\%LUDOX2 & 0.802 & 6.55 & $0.690 \pm 0.007$ & $5.6 \pm 0.2$ \\
\hline SD250S & 1 & 6.55 & 1 & $3.0 \pm 0.1$ \\
\hline SD250S/0.5\%LUDOX3 & 0.983 & 6.55 & $0.901 \pm 0.015$ & $6.9 \pm 0.4$ \\
\hline SD250S/1.0\%LUDOX3 & 0.967 & 6.55 & $0.881 \pm 0.016$ & $5.1 \pm 0.4$ \\
\hline SD250S/1.5\%LUDOX3 & 0.950 & 6.55 & $0.876 \pm 0.018$ & $4.2 \pm 0.5$ \\
\hline
\end{tabular}



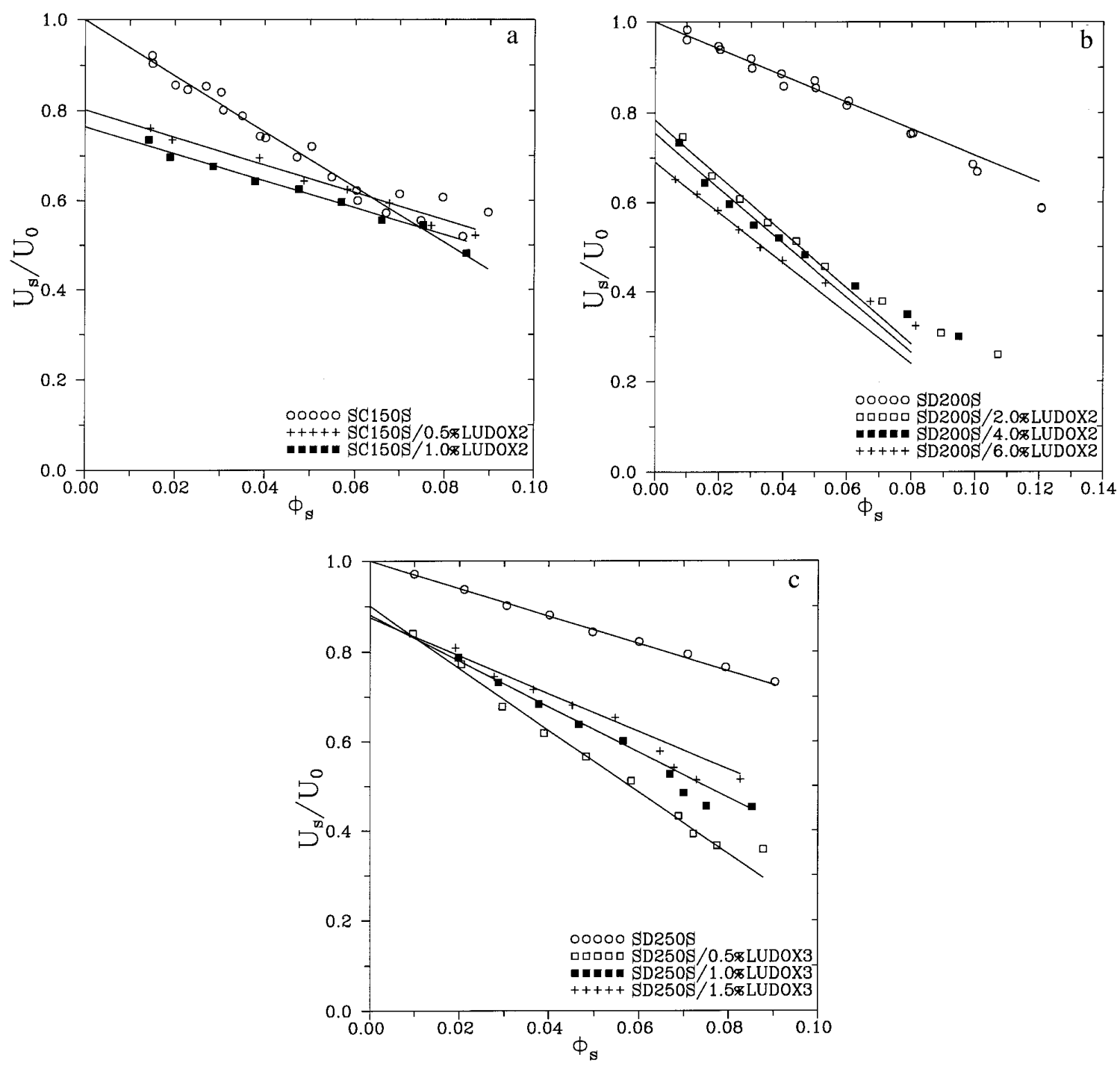

FIG. 4. The sedimentation velocity $U_{\mathrm{s}} / U_{0}$ of large silica spheres as a function of the silica sphere volume fraction $\phi_{\mathrm{s}}$ for the three silica systems with various ludox concentrations for (a) SC150S/LUDOX2, (b) SD200S/LUDOX2, and (c) SD250S/LUDOX3. The solid lines are best fits to Eq. [9] and the used values for $P\left(=1+S_{\mathrm{sl}} \phi_{1}\right)$ and $K$ are given in Table 3. The experimental results of the monodisperse silica systems are also given. The first addition of ludox drastically reduces the sedimentation velocity of the silica spheres, which is caused by adsorption of ludox on the silica spheres. Further additions of ludox only result in small reductions of the sedimentation velocity in accordance with Batchelor's predictions (Eq. [9]).

layer of smaller spheres has not been calculated as far as we know. To explain our results at least qualitatively, we note that the surface layer can be seen as a two-dimensional "porous medium", of small, fixed spheres (Fig. 5). The high surface area and the hydrodynamic interactions in this medium will increase the drag at already fairly low densities. (That is, low surface coverage of ludox spheres.) As an upperestimate of this effect, assume that the adsorbed ludox particles immobilize the liquid between them. The friction of the composed particle is then be approximated by

$$
f_{\text {comp }} \approx 6 \pi \eta\left(a_{\mathrm{s}}+2 a_{1}\right),
$$

where $f=6 \pi \eta a_{\mathrm{s}}$ is the friction of the bare silica sphere. For the used particle mixtures $f_{\text {comp }}$ is about $14-24 \%$ larger than $f$. This is also the order of magnitude of the observed reduction of the sedimentation velocity. It should be noted that such a reduction will only occur if the surface coverage is sufficiently low. In the case of a dense layer of ludox particles, the mass increase will dominate the increase in the friction, and the particles will sediment faster. The friction increment will only dominate in the case of a small number of ludox particles.

Further additions of ludox only cause small decreases in the sedimentation velocity, as can be seen in Fig. 4. The DLS results, described in Section 4.3., show that the number of adsorbed ludox particles per silica sphere is less than (about) 60. Because in the dispersions after the first ludox addition, the ratio of the number of ludox particles and the 


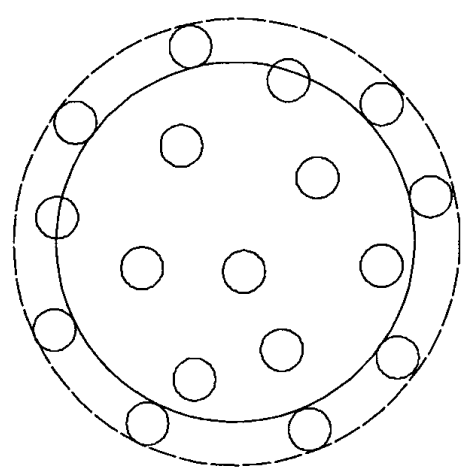

FIG. 5. Schematic representation of a silica sphere with adsorbed small ludox particles. The dashed circle indicates the effective particle size for the friction. This enhanced friction dominates the mass increase, so sedimentation is retarded in comparison with a bare sphere.

number of silica spheres is at least 200, all available adsorption sites on the silica surface probably are occupied. Further addition of ludox then mainly increases the number of free ludox particles and thus $\phi_{1}$. According to Eq. [5] together with the viscosity results of LUDOX3 as described in 4.2.1., and the density ratio $\gamma$ for the used mixtures, the reduction of $P$ is expected to be only a few percent. This reduction of $P$ roughly equals the predictions by Batchelor (see Table 3 ). Moreover, it explains the observed small decreases in the sedimentation velocity. So, apart from the adsorption of ludox on silica, the resulting particles settle more slowly because of the hydrodynamic interactions between the (free, nonadsorbed) small and large spheres as predicted by Batchelor.

4.2.2. The change in $K$. As can be seen in Fig. 4 and Table 3 the slope $K$ changes also when ludox is added. After the first addition of ludox, for both the SD200S and SD250S particles, the slope $K$ changes from 3.0 to, respectively, 6.3 \pm 0.4 and $6.9 \pm 0.4$, which can be understood as follows. Due to the adsorbed ludox on the silica spheres, the distance of closest approach between two silica spheres increases. It can be calculated that only 6-12 ludox particles per silica sphere are needed to prevent two silica spheres from touching. So, due to the adsorbed ludox particles the silica spheres remain always at a larger surface-to-surface distance than 3 $\mathrm{nm}$, which stems from the thickness of twice the stearyl layer. The Van der Waals attraction can now be calculated with Eq. [8] with $H$ the radius of a ludox sphere $(\sim 16-19$ $\mathrm{nm}$ ). The result is a contact attraction of less than $-0.12 k_{\mathrm{B}} T$, with the conclusion that the adsorbed ludox particles screen the Van der Waals attractions between the silica spheres effectively.

The resulting slopes $K=6.3$ and 6.9, which are close to Batchelor's hard-sphere result of $K=6.55$, suggest that the silica spheres (with adsorbed ludox particles) indeed no longer attract each other. One could say that the ludoxsilica interaction increases the effective repulsion between the large silica spheres. Here an analogy can be drawn with solvation forces, induced by an attraction between a solvent component and dispersed particles, which also generates a repulsion between the particles (20). In our case, the "solvent molecules" are small ludox particles. The affinity of "solvent" for the surface is due to roughness of that very surface. However, the origin of the affinity is not relevant (see also Ref. (20)).

The slope for the SC150S particles decreases from $K=$ 6.2 to $K=3.0 \pm 0.3$ after the first ludox addition, which suggests the development of attractions between the initially repulsive SC150S particles. In this case we do not have an explanation for any attractions.

After the first ludox addition the slope $K$ remains (almost) constant for the SC150S/LUDOX2 and SD200S/LUDOX2 systems. Apart from the adsorption, the ludox spheres do not influence the coefficient $K$ but only slightly change $P$. The fact that $K$ remains constant is in accordance with Batchelor's predictions ( see Table 3 ).

For the largest particles, SD250S, the slope $K$ decreases with increasing ludox concentration, which indicates the presence of attractions. Attractions between large spheres in bidisperse systems can be the result of depletion forces (7). When the surface-to-surface distance of two large spheres becomes smaller than the diameter of the small particles, the osmotic pressure of the small particles in the dispersion tends to push the two large spheres together. The result is an attraction between the large spheres, which at contact has, for a large size ratio, the value (7)

$$
V=-\frac{3}{2} k_{\mathrm{B}} T \frac{R_{\text {large }}}{R_{\text {small }}} \phi_{\text {small }},
$$

with $R$ the particle radius. For the SD250S/LUDOX3 mixture with the highest ludox concentration this energy is about $-0.35 k_{\mathrm{B}} T$. However, adsorption of ludox particles will suppress or even eliminate this depletion force, because the large spheres can not approach another closely enough. It should be mentioned that in the case the depletion forces play a role, Eq. [4] should be extended with a term of order $\phi_{i} \phi_{j}$. This term accounts for the fact that the coefficient $S_{i i}$ contains a contribution proportional to $\phi_{j}$.

An alternative attraction is the bridging of the silica spheres by the ludox particles leading to a slow coagulation.

\subsection{Adsorption of Ludox on Silica}

4.3.1. Verification of adsorption. The drastic reduction in the sedimentation velocity $U_{\mathrm{s}}$ after addition of ludox spheres prompted us to study the possible adsorption of ludox on silica in more detail. After all, alternative explanations for the observed sedimentation results, such as local density inhomogeneities, can be given in the form of a relatively large amount of small ludox spheres in the direct 


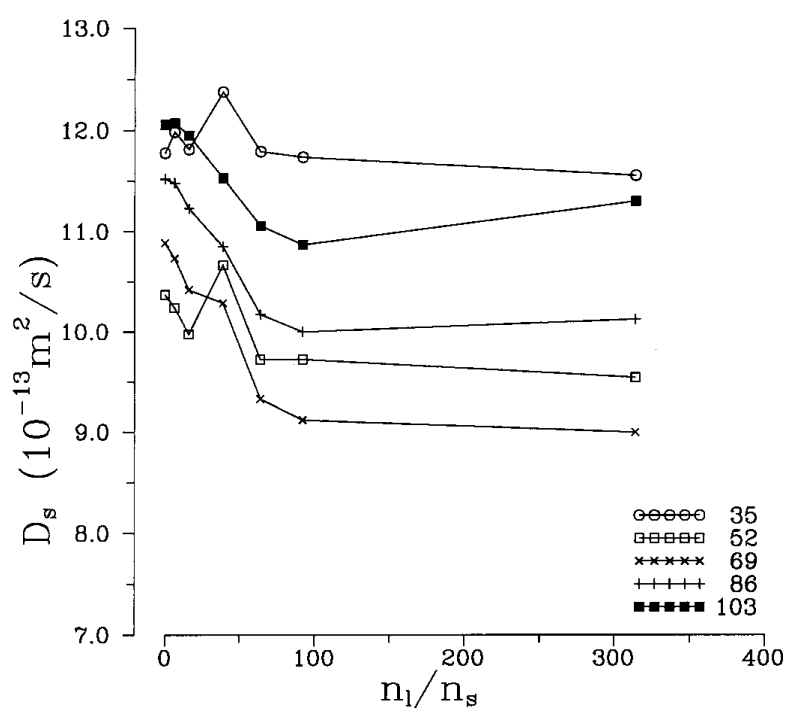

FIG. 6. Diffusion coefficients $D_{\mathrm{s}}$ as a function of the ratio of the number $n_{1}$ of LUDOX3 particles and the number $n_{\mathrm{s}}$ of SD200S spheres in the sample for five scattering angles between $35^{\circ}$ and $103^{\circ}$. The reduction of $D_{\mathrm{s}}$ of $3-$ $13 \%$ for each angle with increasing $n_{1} / n_{\mathrm{s}}$ is caused by adsorption of ludox on silica.

vicinity of the large silica spheres, which retard the silica spheres due to a local high viscosity. Three experiments have been performed to assess whether or not ludox particles are fixed on the silica surface.

With dynamic light scattering a diffusion coefficient $D$ is measured, related for monodisperse, free particles to the particle friction as $D=k_{\mathrm{B}} T / f$ (see Eq. [6]). The same hydrodynamic friction appears in the sedimentation equations (Eq. [1]). So, analogous to the sedimentation velocity, the measured diffusion coefficient of silica should show a strong reduction due to the adsorption of ludox. Measurements were done on mixtures of SD200S and LUDOX3, with fixed $\phi_{\mathrm{s}}=0.007$ and $\phi_{1}$ increasing from 0 to 0.003 . The scattered intensity $I$ is proportional to $n V_{\mathrm{p}}^{2}$ (where $n$ is the particle number density and $V_{\mathrm{p}}$ is the particle volume). For the used particles in the above-mentioned concentration ranges of ludox (1) and silica (s) it turns out that $n_{1} V_{\mathrm{p}, 1} / n_{\mathrm{s}} V_{\mathrm{p}, \mathrm{s}}$ $\ll 1$, so the scattered intensity due to the ludox spheres can be neglected. The measured autocorrelation functions were fit with a second-order cumulant method to obtain the diffusion coefficient of the silica $D_{\mathrm{s}}$ at five scattering angles between $35^{\circ}$ and $103^{\circ}$. In Fig. 6 the obtained values for $D_{\mathrm{s}}$ are given as a function of $n_{1} / n_{\mathrm{s}}$ for each scattering angle. (The angular dependence probably is due to polydispersity.) Although we cannot account for all absolute values for $D_{\mathrm{s}}$ it is clear that $D_{\mathrm{s}}$ decreases (and thus the friction increases) about $3-13 \%$ at each scattering angle when $n_{1} / n_{\mathrm{s}}$ increases from 0 to about 60 . Because viscosity effects can be neglected for the used low volume fractions (see Section 4.1.2), the reduction of $D_{\mathrm{s}}$ in the range $n_{\mathrm{l}} / n_{\mathrm{s}}=0-60$ must be due to the adsorption of an amount $(<60)$ of ludox parti- cles per silica sphere. The observed reduction in $D_{\text {s }}$ can be compared with the sedimentation results of SD200S mixed with LUDOX2 (with a diameter almost equal to that of LUDOX3 ). Extrapolating the sedimentation results to $\phi_{1} \approx$ 0.003 results in a reduction in $U_{\mathrm{s}}$ of about $10-14 \%$, which qualitatively agrees with the DLS results.

The sediment volumes of several mono- and bidisperse samples were determined in order to find the volume fraction of large silica spheres, $\phi_{\mathrm{s}, \mathrm{sed}}$, in the sediments. If the ludox particles are fixed on the silica spheres, they will increase the volume of the sediment and hence decrease $\phi_{\mathrm{s}, \mathrm{sed}}$. If the ludox particles can freely move, they are situated in the interstices between the silica spheres or they are expelled from the sediment, and $\phi_{\mathrm{s} \text { sed }}$ will hardly differ from the monodisperse silica volume fraction.

Sediments were formed in separate dispersions of the two largest silica spheres, and mixtures of each of these spheres with LUDOX3 particles. The height of the sediment was followed in time until it reached a constant value. The used amounts of LUDOX3 particles were so small that in the case where no adsorption takes place, they would not increase the volume of the sediment significantly. The results, given in Table 4 , show that $\phi_{\mathrm{s} \text {,sed }}$ of the pure silica systems is higher than $\phi_{\mathrm{s}, \text { sed }}$ of the bidisperse systems. The differences in $\phi_{\mathrm{s}, \mathrm{sed}}$ demonstrate that the surfaces of the silica spheres are kept at some distance in binary sediments. The only plausible origin of this phenomenon is that the small spheres stick at the silica surface and hence induce an expansion of the silica sediment.

The third experiment to verify the presence of adsorbed ludox was performed as follows. After (repeated) removal of free ludox spheres from a mixture of silica and ludox, the sedimentation velocity $U_{\mathrm{s}}$ of the silica was measured. If an amount of ludox particles is adsorbed on the silica surface, the measured $U_{\mathrm{s}}$ should remain much lower than the value for the bare silica spheres. If the ludox particles do not adhere to the silica surface, the "bare" silica spheres are recovered and the measured $U_{\mathrm{s}}$ should equal the value in 4.1.1.

$U_{\mathrm{s}}$ was determined for two mixtures of SD200S and LUDOX3. Then the tubes were left undisturbed to allow the silica spheres to settle. After two weeks the supernatant dispersion (containing LUDOX3 and cyclohexane) above the

TABLE 4

Silica Volume Fractions in Sediments $\phi_{\mathrm{s}, \text { sed }}$ of Pure Silica and of Mixtures of Silica and Ludox

\begin{tabular}{lc}
\hline \multicolumn{1}{c}{ System } & $\phi_{\text {s,sed }}$ \\
\hline SD200S & $0.61 \pm 0.01$ \\
SD200S/LUDOX3 & $0.55 \pm 0.02$ \\
SD250S & $0.63 \pm 0.02$ \\
SD250S/LUDOX3 & $0.58 \pm 0.01$ \\
\hline
\end{tabular}




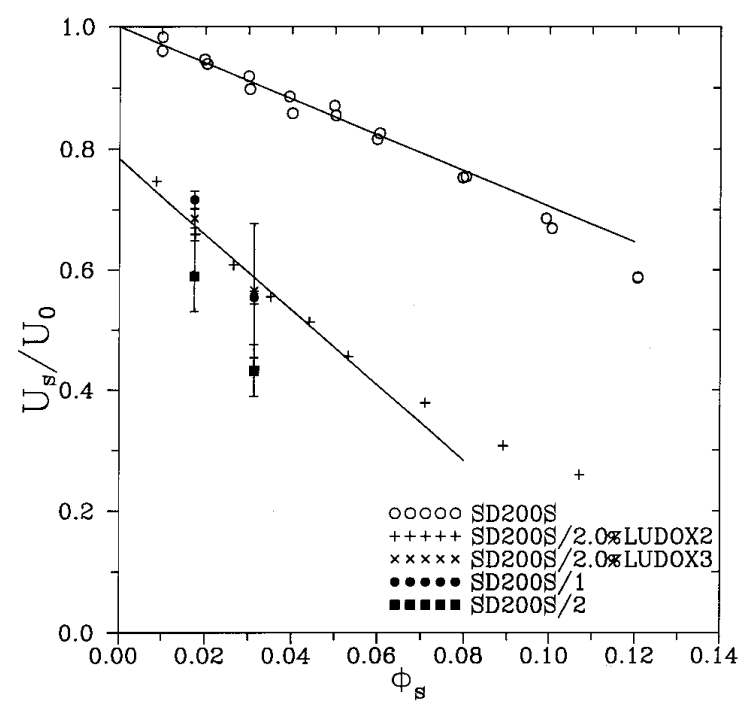

FIG. 7. $U_{\mathrm{s}}$ as a function of $\phi_{\mathrm{s}}$ for silica spheres SD200S without any ludox added (O), SD200S/2.0\%LUDOX2 (+), and SD200S/LUDOX3 $(\times)$. Filled circles indicate the values for $U_{\mathrm{s}}$ after the first exchange of (a large part of) the ludox dispersion for cyclohexane. Filled squares indicate the values after the second exchange. The solid lines are best fits of the results of SD200S and SD200S/2.0\%LUDOX2 from Fig. 4b. It is clear that once ludox is added to a silica dispersion, adsorbed ludox particles cannot be removed. The values for $U_{\mathrm{s}}$ remain below the values for the "bare", silica systems $(\bigcirc)$, demonstrating that the ludox particles must be fixed on the silica surface.

sediment was removed and replaced by pure cyclohexane. After homogenization $U_{\mathrm{s}}$ was measured again. After complete settlement of the silica particles again the dispersion above the sediment was replaced by cyclohexane and $U_{\mathrm{s}}$ was measured. The sedimentation results (see Fig. 7) clearly show that the sedimentation velocity remains very low in comparison with the obtained values for the SD200S dispersion of "bare" silica spheres. A sample of the dispersion (after two ludox "extractions"') was studied with TEM. On representative micrographs, as in Fig. 8a, no free small particles can be found, only small particles touching the large silica spheres. If ludox particles would not stick, the extraction procedure would yield a ludox volume fraction in the final dispersion, studied with TEM, of at most $2 \times$ $10^{-4}$. This corresponds to a maximum of about four ludox particles per silica sphere. On micrographs, however, much more ludox particles per silica sphere can be seen. This confirms that one can not extract the small spheres from the mixture.

4.3.2. Origin of the adsorption. All control experiments clearly confirm the presence of (according to DLS less than about 60) ludox particles adsorbed on a Stöber silica sphere.

Are specific sites on the large silica spheres responsible for the adsorption of the small ludox particles or do the ludox particles adsorb at any location on the silica surface? In the case of a completely nonspecific adsorption, the ludox particles would induce coagulation, because they would form connections between the large spheres. In the case of a highly specific adsorption (like lock and key) each silica sphere can only be connected to one ludox particle. In the realistic intermediate case of several sites per silica sphere available for ludox adsorption, at most a slow coagulation will be possible. However, we do not observe any flocculation of the large spheres (on a timescale of days). This would cause an increase in the sedimentation velocity $U_{\mathrm{s}}$ instead of the observed decrease. This observation indicates that an excess amount of ludox is present in the dispersion such that all adsorption sites are quickly occupied by ludox particles. Then there are no sites left for a ludox sphere to link two silica spheres (not even on a large time scale). Only for the largest silica system SD250S, mixed with LUDOX3, slow coagulation might play a role, because for these samples a small increase in $U_{\mathrm{s}}$ is found.

The number of ludox particles which can be placed on a silica surface can roughly be estimated with the so-called random parking method. Feder (21) found with computer simulations that randomly positioned, nonoverlapping disks can only occupy $55 \%$ of a flat surface. Applying these results to sphere mixtures with a large size ratio implies that the number of adsorbed ludox particles per silica sphere will not exceed the range 150-460. From the DLS results an amount of adsorbed ludox particles smaller than 60 was found. The conclusion is that adsorption only occurs on a limited number of attractive patches on the large silica sphere.

The origin of the adsorption is very likely the Van der Waals attraction. For two unequal, smooth spheres at very small distance $H$, the attraction energy would be (18)

$$
V=-\frac{A a_{\mathrm{s}} a_{1}}{6 H\left(a_{\mathrm{s}}+a_{1}\right)}
$$

For particles with a dense stearyl coating the distance of closest approach is $H=3 \mathrm{~nm}$, which results in attractions between the used ludox and silica spheres smaller than $V=$ $-0.15 k_{\mathrm{B}} T$. This very likely underestimates the ludox adsorption energy. It is known, for example, that there are differences in grafting density of the stearyl chains between different particle systems (16). Incomplete coverage of the silica spheres with $\mathrm{C}_{18}$ chains allows the ludox particles to approach them more closely. For example, for values of $H$ of $0.4 \mathrm{~nm}$ the attraction becomes about $-1.0 k_{\mathrm{B}} T$, which still is a modest value. A more important factor for adsorption is the following. On the electron micrograph, used to determine $a_{\text {TEM }}$, the silica surfaces seem to be very smooth. However, at much larger magnifications it can be seen that these spheres show surface irregularities on a nanoscale (see Fig. 8b), which were also observed by Van Helden et al. (16). These irregularities can increase the contact area between a silica and a ludox sphere (as is sketched in Fig. 8c) and 

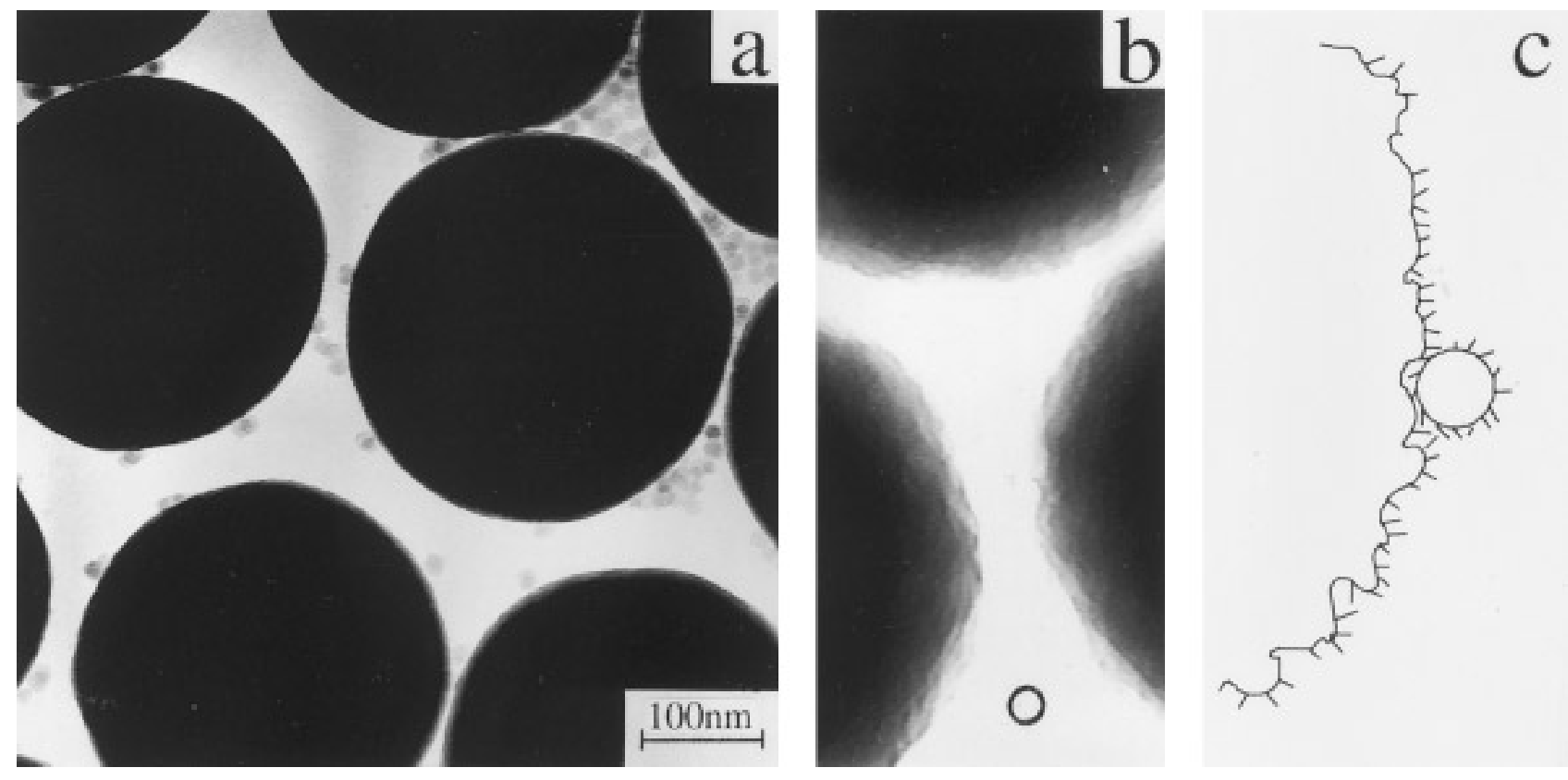

FIG. 8. (a) Transmission electron micrograph of a SD200S/LUDOX3 mixture after removal of free ludox particles. On account of the (estimated) amounts of SD200S and LUDOX3 particles in this dispersion, it is confirmed that one cannot extract the small spheres from a mixture. (b) Electron micrograph of a SC150S particle at a very large magnification, which clearly shows that the particle has surface irregularities on a nanoscale. A typical ludox particle is sketched to illustrate that its radius of about $16 \mathrm{~nm}$ is comparable to the surface irregularities of the large silica spheres. (c) A schematic representation of the possible close approach of a ludox and a silica sphere as a result of surface irregularities on the silica surface and differences in grafting density of the $\mathrm{C}_{18}$ chains.

hence significantly increase the Van der Waals attractions. A rough estimate of the contact area is $2 \pi a_{1}^{2}$. For two parallel flat plates at a distance of about $1.5 \mathrm{~nm}$ such an area would correspond to an attractive energy of $V=-2.8 k_{\mathrm{B}} T$. This estimate gives some idea of the increase in contact attraction experienced by a small ludox particle because of surface irregularities. These irregularities are small in comparison with the silica sphere size. For this reason they do not enhance the attraction between the large spheres.

Actually, for large spheres one would expect that these irregularities decrease the attraction, because the surface-tosurface distance increases. This phenomenon was studied theoretically by Czarnecki and Itschenskij (22). The present work shows that their approach, which predicts a diminished attraction also for unequal spheres, is not valid when one of the interacting particles is very small.

A simple calculation shows that available sites will be quickly occupied. The collision frequency $J$ of small ludox spheres (with a diffusion coefficient $D_{1}$ ) with one large, fixed silica sphere of radius $a_{\mathrm{s}}$ is given by the stationary diffusion flux

$$
J \approx 4 \pi n_{1} D_{1} a_{\mathrm{s}}
$$

where $n_{1}$ is the number density of the ludox spheres. In comparison with a ludox sphere, the diffusion of a silica sphere can be neglected due to the relatively high friction.
With $\phi_{1}=(4 / 3) \pi a_{1}^{3} n_{1}$ and $D_{1}=k_{\mathrm{B}} T / 6 \pi \eta_{0} a_{1}$, Eq. [13] becomes for $T=298 \mathrm{~K}$

$$
J \approx 6.9 \cdot 10^{8} \frac{a_{\mathrm{s}}}{a_{1}^{4}} \phi_{1},
$$

with $a_{\mathrm{s}}$ and $a_{1}$ in $\mathrm{nm}$. Using the particle characteristics SC150S/LUDOX2 mixture, $J \approx 1.4 \times 10^{6}$ which means that for $\phi_{1}=0.005$ every second 7000 ludox spheres collide with a silica sphere. Thus 60 adsorption sites on the silica spheres will be rapidly occupied by ludox. A silica sphere with a ludox particle on its surface has a small diffusion coefficient in comparison with a free ludox particle. So any bridging of silica spheres by a ludox particle will be a relatively slow process.

It can be concluded that the combination of an increased contact area and a reduced distance of closest approach (due to differences in grafting density) leads to Van der Waals attractions of sufficient strength to stick a silica and ludox sphere together. This explanation is consistent with the fairly small number of adsorbed ludox particles, because the number of sufficiently attractive "parking places" will be limited. The interaction between small particles and surface irregularities of larger spheres probably also accounts for the unexplained attractions, which manifested themselves in viscosity measurements (10), small-angle neutron scattering 
(11), and phase separation (8) experiments on mixtures of uncharged silica spheres, which were also coated with stearyl alcohol.

\section{CONCLUSIONS}

Addition of small amounts of small nonsedimenting ludox particles to the Stöber silica dispersions results in a marked reduction of the sedimentation velocity of the large silica spheres. This reduction is much larger than expected from Batchelor's theory for bidisperse, hard spheres. At very low small-particle concentrations, the decrease in settling velocity of the large spheres is due to adsorption of small ludox particles. This adsorption is caused by surface irregularities of the large spheres. Due to the limited number of suitable attractive patches, and thus ludox particles, on the silica surface the friction increment per silica particle dominates the mass increase and thus the sedimentation velocity decreases. This adsorption also screens effectively any (weak) Van der Waals attraction between silica spheres, because the adsorbed ludox particles prevent two silica spheres to touch. (In this respect the adsorption mimics a solvation force (20).)

Further additions of ludox only cause a small additional decrease of the large-sphere sedimentation velocity, mainly because of a viscosity increase. This result is in accordance with Batchelor's calculations. Apart from the observed adsorption phenomenon, Batchelor's theory for the sedimentation of large spheres through a dispersion of small spheres explains the experimental results for colloidal sphere mixtures.

\section{ACKNOWLEDGMENTS}

Carlos van Kats is gratefully acknowledged for performing the main part of the experimental work. Jacques Suurmond is thanked for doing the UC measurements. This work was supported by the Netherlands Foundation for Chemical Research (SON) with financial aid from the Netherlands Organization for Scientific Research (NWO).

\section{REFERENCES}

1. Batchelor, G. K., J. Fluid Mech. 52, 245 (1972).

2. Kops-Werkhoven, M. M., and Fijnaut, H. M., J. Chem. Phys. 74, 1618 (1981).

3. Kops-Werkhoven, M. M., Pathmamanoharan, C., Vrij, A., and Fijnaut, H. M., J. Chem. Phys. 77, 5913 (1982).

4. Buscall, R., Goodwin, J. W., Ottewil, R. H., and Tadros, Th. F., J. Colloid Interface Sci. 85, 78 (1982).

5. (a) Batchelor, G. K., J. Fluid Mech. 119, 379 (1982); (b) Batchelor, G. K., and Wen, C.-S., J. Fluid Mech. 124, 495 (1982).

6. Davis, R. H., and Birdsell, K. H., AIChE J. 34, 123 (1988).

7. Vrij, A., Pure Appl. Chem. 48, 471 (1976).

8. van Duijneveldt, J. S., Heinen, A. W., and Lekkerkerker, H. N. W., Europhys. Lett. 21, 369 (1993).

9. Kaplan, P. D., Rouke, J. L., Yodh, A. G., and Pine, D. J., Phys. Rev. Lett. 72, 582 (1994).

10. Woutersen, A. T. J. M., and de Kruif, C. G., J. Rheol. 37, 681 (1993).

11. Duits, M. H. G., May, R. P., Vrij, A., and de Kruif, C. G., J. Chem. Phys. 94, 4521 (1991).

12. Jansen, J. W., de Kruif, C. G., and Vrij, A., J. Colloid Interface Sci. 114, 501 (1986).

13. Thies-Weesie, D. M. E., Philipse, A. P., Nägele, G., Mandl, B., and Klein, R., J. Colloid Interface Sci. in press.

14. Einstein, A., "Investigations on the Theory of Brownian Movement'" (R. Fürth, Ed.). Dover, New York, 1956.

15. Stöber, W., Fink, A., and Bohn, E., J. Colloid Interface Sci. 26, 62 (1968).

16. van Helden, A. K., Jansen, J. W., and Vrij, A., J. Colloid Interface Sci. 81, 354 (1981).

17. Pathmamanoharan, C., and Philipse, A. P., J. Colloid Interface Sci. 165, 519 (1994).

18. Israelachvilli, J. N., “'Intermolecular and Surface Forces.' Academic Press, London, 1978.

19. Jansen, J. W., de Kruif, C. G., and Vrij, A., J. Colloid Interface Sci. 114, 471 (1986).

20. Penders, M. H. G. M., and Vrij, A., Prog. Colloid Polym. Sci. 89, 1 (1992).

21. Feder, J., J. Theor. Biol. 87, 237 (1980).

22. Czarnecki, J., and Itschenskij, V., J. Colloid Interface Sci. 98, 590 (1984). 